\title{
Intensified environmental surveillance supporting the response to wild poliovirus type 1 silent circulation in Israel, 2013
}

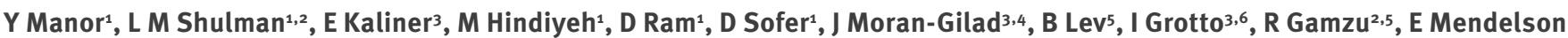

(ellamen@sheba.health.gov.il),

1. Central Virology Laboratory, Ministry of Health, Chaim Sheba Medical Center, Tel Hashomer, Israel

2. School of Public Health, Sackler Faculty of Medicine, Tel Aviv University, Tel Aviv, Israel

3. Public Health Services, Ministry of Health, Jerusalem, Israel

4. European Society of Clinical Microbiology and Infectious Diseases (ESCMID) Study Group for Molecular Diagnostics (ESGMD)

5. Directorate, Ministry of Health, Jerusalem, Israel

6. Department of Public Health, Faculty of Health Sciences, Ben Gurion University of the Negev, Beer Sheva, Israel

Citation style for this article:

Manor Y, Shulman LM, Kaliner E, Hindiyeh M, Ram D, Sofer D, Moran-Gilad J, Lev B, Grotto I, Gamzu R, Mendelson E. Intensified environmental surveillance supporting the response to wild poliovirus type 1 silent circulation in Israel, 2013. Euro Surveill. 2014;19(7):pii=20708. Available online: http://www.

eurosurveillance.org/ViewArticle.aspx?Articleld=20708

An emergency response was triggered by recovery of wild poliovirus type 1 (WPV1) of the South Asia (SOAS) lineage from sewage in southern Israel in April 2013 during routine environmental surveillance. Public health risk assessment necessitated intensification of environmental surveillance in order to facilitate countrywide monitoring of WPV1-SOAS circulation. This involved increasing sampling frequency and broadening the geographical area, for better coverage of the population at risk, as well as modifying sewage testing algorithms to accommodate a newly developed WPV1-SOAS-specific quantitative real-time RT-PCR assay for screening of RNA extracted directly from sewage concentrates, in addition to standard virus isolation. Intensified surveillance in 74 sites across Israel between 1 February and 31 August 2013 documented a sustained high viral load of WPV1-SOAS in sewage samples from six Bedouin settlements and two cities with Jewish and Arab populations in the South district. Lower viral loads and intermittent detection were documented in sampling sites representing 14 mixed communities in three of the five health districts in central and northern Israel. Environmental surveillance plays a fundamental role in routine monitoring of WPV circulation in polio-free countries. The rapid assay specific for the circulating strain facilitated implementation of intensified surveillance and informed the public health response and decision-making.

\section{Introduction}

In the drive towards global eradication of poliomyelitis, as of 2013, only three countries remained endemic for wild-type poliovirus (WPV) infection: Afghanistan, Pakistan and Nigeria, with recent introduction of the virus resulting in paralytic cases to countries with suboptimal immunisation coverage in Africa (Somalia, Kenya, Ethiopia and Cameroon) and in Asia (Syria) [1]. Only WPV type 1 (WPV1) is currently circulating, consisting of two major lineages: the South Asia (SOAS) lineage, which is indigenous to Pakistan and Afghanistan, and the West Africa (WEAF) B lineage, which is indigenous to Nigeria [2].

Poliovirus circulation in highly immune populations is far less likely to be detected via identification of clinical paralytic poliomyelitis cases, which are expected to be very rare or absent (silent circulation), and thus environmental surveillance for poliovirus has become a very useful tool for population-based alert and monitoring of WPV activity. This approach has been implemented by several countries, both for early detection of WPV introduction and transmission as well as for detection of vaccine-derived neurovirulent polioviruses (VDPVs) that emerge following the use of oral poliovirus vaccines (OPVs) [3,4].

Israel has been free of poliomyelitis since the last outbreak caused by WPV 1 in 1988 , which resulted in 15 paralytic cases $[5,6]$. The outbreak strain originated in Egypt and arrived in 1987 from Gaza to Rahat and spread in 1988 from Rahat to central Israel. A routine environmental surveillance for poliovirus programme has been implemented since then in Israel, Gaza and the West Bank, which monitors sentinel sites that represent large populations (such as the Shaf-Dan, a sewage treatment facility in the metropolitan Tel Aviv area) and populations (such as Rahat) considered at high risk for introduction of WPV from other countries. A combined inactivated poliovirus vaccine (IPV)/trivalent oral poliovirus vaccine (tOPV) routine vaccination schedule was in place until 2005, when it was replaced by an IPV-only schedule [13].

Over the years, the environmental surveillance programme has detected several introductions of WPV into Gaza and Israel, but subsequent circulation in the 
local community occurred only once in Gaza in 1994-45 [7-9]. In addition, it has detected two lineages of highly diverged type 2 VDPV in the Tel Aviv sewage system, excreted by single individuals [9-11], demonstrating the high sensitivity of environmental surveillance for monitoring large populations. Laboratory methods for sample treatment and poliovirus isolation, including the plaque formation approach, are also a major factor in the sensitivity of the environmental surveillance, as reviewed by Hovi et al. [3]. Plaque formation allows a rough estimation of the virus circulation intensity since the number of viral plaque-forming units (PFU) present in the original sewage sample can be deduced based on spiking experiments [8].

In December 2012, WPV1-SOAS was detected in sewage collected from Cairo, Egypt [11], where systematic environmental surveillance for poliovirus has been in place since 2000. A large immunisation campaign initiated as a response led to the disappearance of the virus from the sewage and, by implication, from the population at large [12].

In April 2013, a surge in the number of plaques recovered on L20B cells from a sewage sample collected in Rahat and Beer Sheva, two major cities in southern Israel, occurred. Identification of the plaques as WPV1 suggested an importation and possible circulation of WPV 1 in the region [13]. These alarming findings prompted an urgent assessment and response by the Public Health Services of the Ministry of Health. Notably, intensification of environmental surveillance for poliovirus played a key role in monitoring the spread of the virus. Here we describe the modification and enhancement of the environmental sampling and laboratory methods used in order to meet the increased demand for processing of sewage samples and generation of surveillance data that will inform public health response and incident management.

\section{Methods}

Sampling sites and sample collection

Since 1989, composite sewage samples have been collected monthly from sentinel sites covering $30-40 \%$ of the Israeli population using computerised automatic inline samplers located at the mouth of sewage treatment facilities [8]. These samplers collect and pool aliquots hourly over a 24 -hour period to a final volume of $1 \mathrm{~L}$. Sample collection in sites without automatic inline samplers and at upstream lines (carrying sewage from around the city or from different communities into the main sewage line that enters the sewage treatment facility) was carried out using computerised automatic portable samplers (Sigma SD9oo portable samplers, $\mathrm{HACH}$, Loveland, CO, United States). Samples were transported and analysed at the Ministry of Health Central Virology Laboratory.
Poliovirus purification and isolation from sewage samples

The methods used for sample concentration and virus purification from sewage samples during the routine surveillance period have been previously described in detail [11]. Poliovirus was plaque isolated in L2OB cells $(2 \mathrm{~mL}$ of sample inoculated into four $10 \mathrm{~cm}$ tissue culture plates) and the number of plaques isolated on each plate was recorded. Each plaque was further propagated in tube cultures of Hep2C cells to obtain high amount of virus for identification and characterisation. Plaques from Gaza, the West Bank and Rahat were propagated at $40{ }^{\circ} \mathrm{C}$ to select against Sabin strains originating from OPV, which is included in the Palestinian routine immunisation schedule. These strains were common in Rahat due to family relationships and close contact of Israeli Bedouins with their relatives in Gaza. Plaques from all other sites of Israel were propagated at $37^{\circ} \mathrm{C}$ as OPV strains are very rarely found there because of the exclusive use of IPV in Israel. Supernatants from cultures showing cytopathic effects (CPEs) were then subjected to molecular analysis for virus identification and sequencing [11].

Following the detection of WPV1 in Rahat in April 2013 that failed to grow at $40{ }^{\circ} \mathrm{C}$ and the subsequent implementation of highly intensified countrywide environmental surveillance for poliovirus, laboratory protocols were amended as following: for poliovirus isolation in tissue culture, the $40^{\circ} \mathrm{C}$ selec tive growth of plaquepurified viruses on Hep2 $\mathrm{C}$ tube cultures was replaced with propagation on $\mathrm{L} 20 \mathrm{~B}$ tube cultures at $37{ }^{\circ} \mathrm{C}$. In addition, four L2OB tube cultures were each inoculated with $0.3 \mathrm{~mL}$ aliquots of processed sewage and incubated at $37{ }^{\circ} \mathrm{C}$ for 5 days or until full CPEs developed. This step yielded mass cultures and was performed in parallel to the inoculation of tissue culture plates for plaque formation. The number of CPE-positive tubes was recorded, RNA was extracted from a pool of the CPE-positive culture tubes and was subjected to molecular analysis according to standard protocols of the Central Virology Laboratory [14].

\section{qRT-PCR assay specific for WPV1-SOAS circulating in Israel}

The development and validation of a real-time RT-PCR (qRT-PCR) assay specific for the circulating virus for rapid detection of the virus directly in concentrated sewage samples is described elsewhere [15]. Briefly, two sets of primers and probes were designed based on the viral protein 1 (VP1) sequences of five plaquepurified isolates from sample number 8099 collected on 9 April 2013 in Rahat. The analytical sensitivity and specificity of the assay, as well as its positive predictive value and negative predictive value, were found to be $100 \%$ compared with virus isolation in 50 sewage samples tested, of which $40 \%$ were positive and $60 \%$ were negative, with high correlation of the cycle threshold $(\mathrm{Ct})$ values with the number of plaques obtained for each sample. 
qRT-PCR assays for intratypic differentiation of poliovirus isolates and for non-poliovirus enteroviruses

The Sabin 1,2 and 3 multiplex and pan poliovirus types 1,2 and 3 components of the intratypic differentiation assay developed and provided to the Global Polio Laboratory Network (GPLN) by the United States Centers for Disease Control and Prevention (CDC) was used [16]. The assay identified the presence of WPV 1 and indicated whether vaccine strains of the same serotype or other serotypes were present. In addition, an in-house pan-enterovirus qRT-PCR assay $[14,17]$ was used when polio type-specific primers were not available, for example, for identification of wild poliovirus of unknown type.

\section{Sequence analysis of virus isolates}

Molecular epidemiological and phylogenetic characterisation based on complete VP1 capsid protein sequence for all virus isolates were performed as previously reported [12].

\section{Results}

\section{Detection of SOAS-WPV1 importation to} southern Israel and intensified environmental surveillance

Sewage samples collected in Rahat as part of the routine environmental surveillance in April 2013 yielded 20,800 plaques on $\mathrm{L} 20 \mathrm{~B}$ cells. Sequence analysis identified the virus isolates as WPV 1 belonging to the SOAS lineage, with closest match to the virus isolated from sewage in Egypt in December 2012 [18]. The viral load in the sewage sample was calculated from the number of plaques isolated: by taking into consideration of the size of the sampled population, the number of faecal excretors of the virus could be roughly estimated to be tens or even hundreds of excretors, indicating ongoing indigenous circulation. Although the sample collected in April turned out to be a grab sample (taken at one time point) that yielded an 'off-scale' number of plaques, this finding was still considered alarming. Retrospective examination of all sewage samples from January 2013 revealed 203 plaques of the WPV1-SOAS in a sample collected in Rahat in March, and 3 and 29 WPV1-SOAS plaques in samples collected in Beer Sheva in February and March, respectively.

The investigation and response [13] included highly intensified environmental surveillance, first in other communities in southern Israel and subsequently nationwide. The sampling strategy included mostly sample collection at STFs but on several occasions, particularly when STFs represented separate communities, additional samples were collected from upstream sewage lines by a mobile laboratory operated by the Central Virology Laboratory using portable samplers. The number of samples obtained monthly rose from May 2013 (12 samples), with 47, 57, 145 and 53 samples in June, July, August and September, respectively, and with average weekly numbers of $12-36$ samples.
This increase in demand for sample analysis necessitated the development of new algorithms for sample processing and testing in order to meet the challenge.

\section{Incorporation of a novel sewage-testing algorithm}

A new testing algorithm was implemented in order to cope with the large number of samples and the need for rapid results. It provided the following advantages: firstly, a fourfold increase in the number of sewage samples processed daily, by concentrating $500 \mathrm{~mL}$ of raw sewage instead of $1 \mathrm{~L}$, as done in the routine $E S P$, and recovering the virus in $15 \mathrm{~mL}$ instead of $30 \mathrm{~mL}$ final volume to preserve the concentration factor. This allowed us to overcome the initial bottleneck of largevolume centrifugation and to assign for the process two workers with staggered starting times. Secondly, rapid identification of WPV1-positive samples was possible using a novel approach that included testing of RNA extracted directly from concentrated sewage by a qRTPCR screening assay specific for the circulating virus [15]. Thirdly, inoculation of four $L 20 B$ tube cultures to produce a mass culture of all viruses in the sample, in parallel with the plaque-formation assay, thereby shortened the time for virus molecular analysis if and when indicated. A comparison of the two algorithms is shown in Figure 1. The original algorithm produced results only after 16-23 days by sequence analysis of isolates whereas the modified algorithm produced preliminary WPV1-SOAS positive/negative and qRT-PCR results within 3-4 days, confirmation by intratypic differentiation within 9 days and full sequencing within 10-16 days. This approach allowed us to immediately identify communities at risk and to prioritise samples for further analysis and plan site re-sampling. Thus, all three arms of the new testing algorithm supported each other and allowed a higher throughput (up to 50 sewage samples per week) and shorter turnaround time for results. Notably, the quantification of viral load by the qRT-PCR assay correlated well with the plaque number obtained for each sample, as was found during the validation process of the assay [15].

Poliovirus isolation and identification algorithms employed during routine environmental surveillance (A) and after discovery of WPV1-SOAS (B). The algorithm shown in panel $A$ confirms isolation of any poliovirus type by sequence analysis within 16 to 23 days and allows processing of 6 to 12 samples per week. Quantitative evaluation of plaque number is done after the final identification. The algorithm shown in panel $B$, incorporating additional steps (shown in blue), allows primary detection and quantification of WPV1SOAS by qRT-PCR within 3 to 4 days, confirmation by intratypic differentiation and quantitative evaluation of plaque number within 9 days and full sequence results within 10 to 16 days. It also allows processing of up to 50 samples per week.

Altogether, between early January and the end of August 2013, the Central Virology Laboratory received 
Sewage processing and testing algorithm in routine environmental surveillance for poliovirus (A) and during WPV1-SOAS circulation (B), Israel, 2013

A

Routine environmental surveillance

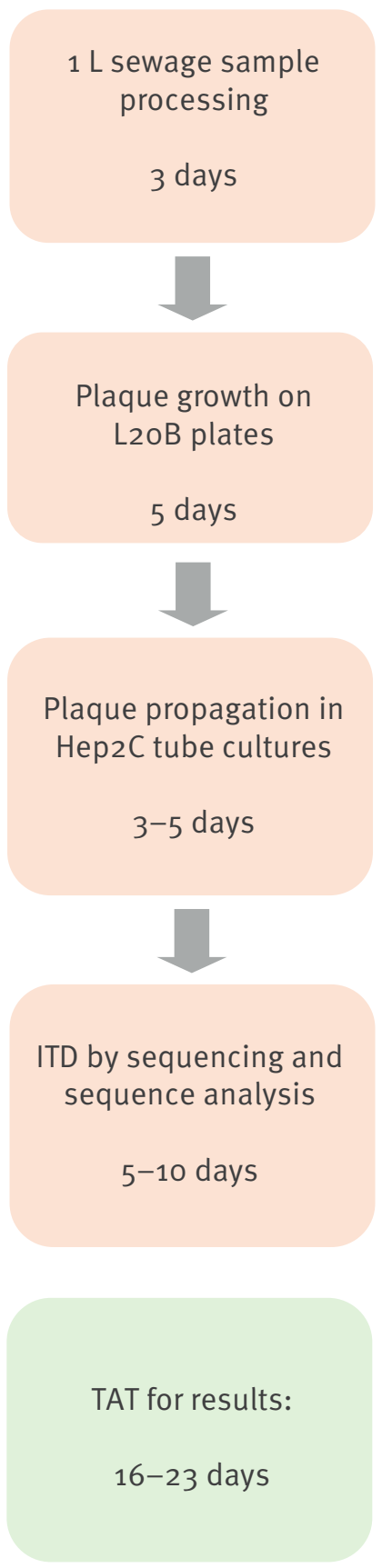

B

During WPV1-SOAS circulation in Israel, 2013

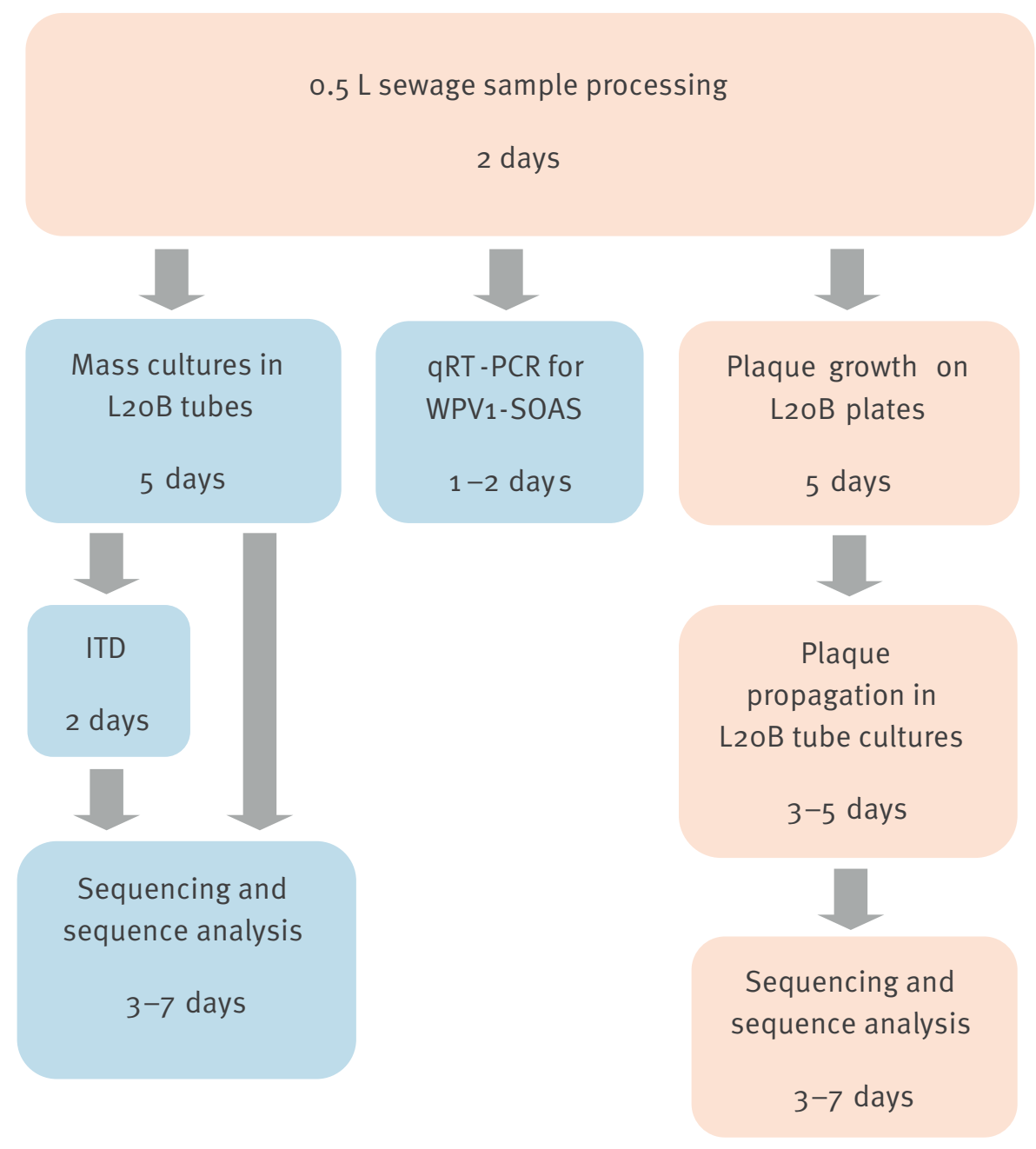

TAT for preliminary (qRT-PCR) results: $3-4$ days

TAT for ITD results: 9 days

TAT for sequencing results: $10-16$ days

ITD: intratypic differentiation; q: quantative; SOAS: South Asia; TAT: turnaround time; WPV1: wild poliovirus type 1. 
Location of sewage sampling sites with WPV1-SOAS testing results in the South, Jerusalem and Centre (part) health districts, Israel, February-August 2013

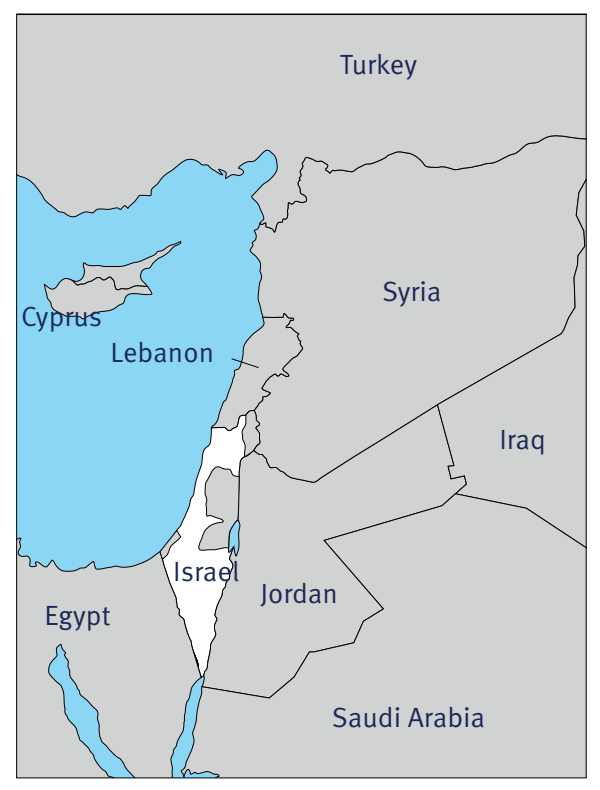

Always positive

A Intermittently positive

Negative

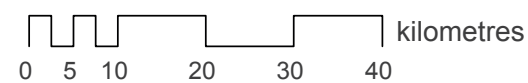

SOAS: South Asia; WPV1: wild poliovirus type 1.

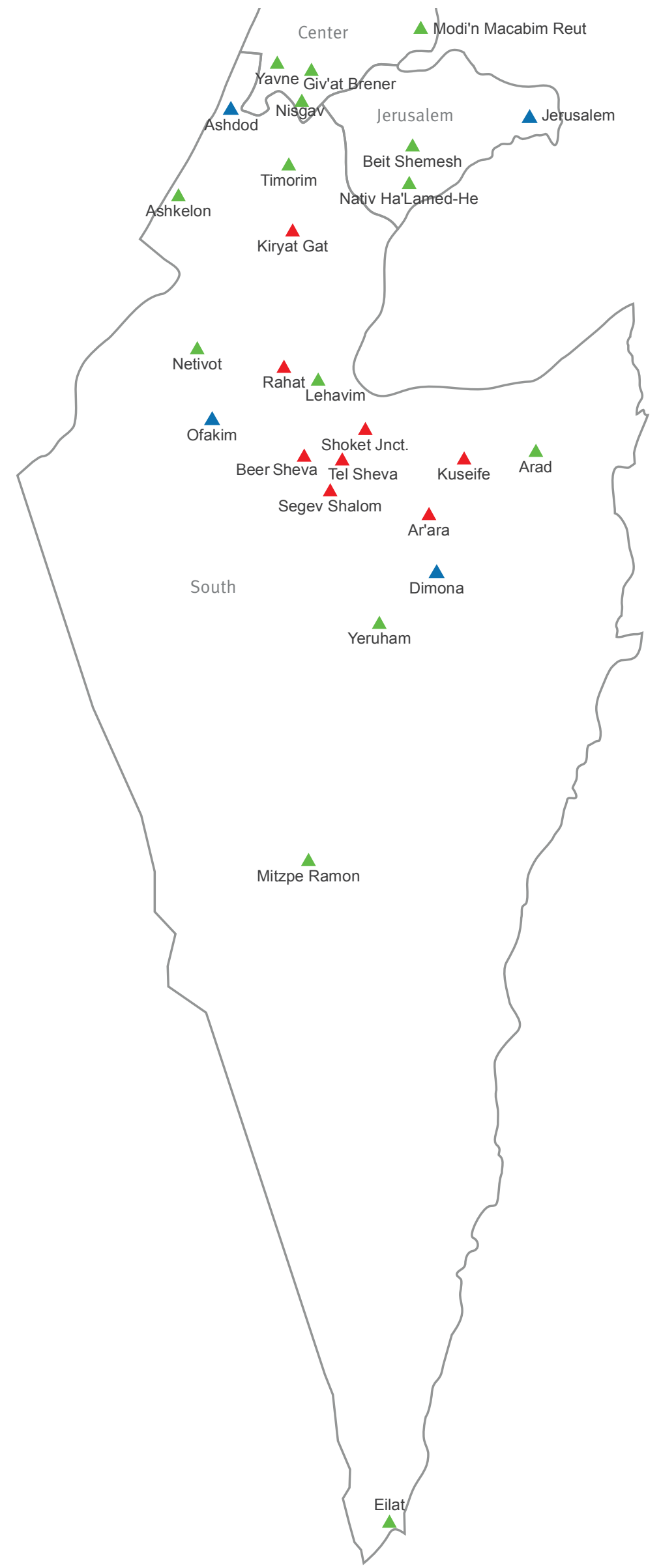


and tested 262 sewage samples, most of them between mid-July and mid-August. These comprised 192 samples obtained from 75 sites across Israel, as well as 70 samples obtained from the Palestinian Ministry of Health.

\section{Circulation of WPV1-SOAS in the South health district}

Of the 69 sites sampled, 23 were in the South health district, with catchment areas covering Bedouin cities and villages, semi-nomadic Bedouin communities, Jewish cities and combinations of Jewish and Bedouin cities or villages. Sampling intervals were one or two weeks for sites belonging to one or more of the following categories: (i) WPV1-SOAS-positive sites; (ii) positive or negative sites from major cities; or (iii) high-risk sites representing populations having close contacts (close family relationships as well as intensive work and social contacts) with the southern
WPV1-SOAS-positive communities. Sites not belonging to any of these categories were sampled once every few weeks or only once. Results were categorised by the relative number of plaques obtained during the study period, as shown in Figure 2, which illustrates the geographical location of 19 of 23 sampling sites in the South health district (excluding two upstream lines of Beer Sheba sewage treatment facility and the Arad sewage treatment facility) and the respective findings. Eight of these sites were persistently positive over the study period according to most samples collected, six covering Bedouin communities, one covering Jewish community (Kiriat Gat) and one covering Jewish and Bedouin communities (Beer Sheva). Three sites covering Jewish communities were intermittently positive (steady or occasional).

A total of 10 sites, eight covering Jewish cities (all shown in Figure 2) and two covering neighbourhoods

\section{FIGURE 3}

Dynamics over time of sewage WPV1-SOAS testing results in three sampling sites ${ }^{a}$ in southern Israel, February-August 2013

Rahat sewage treatment facility

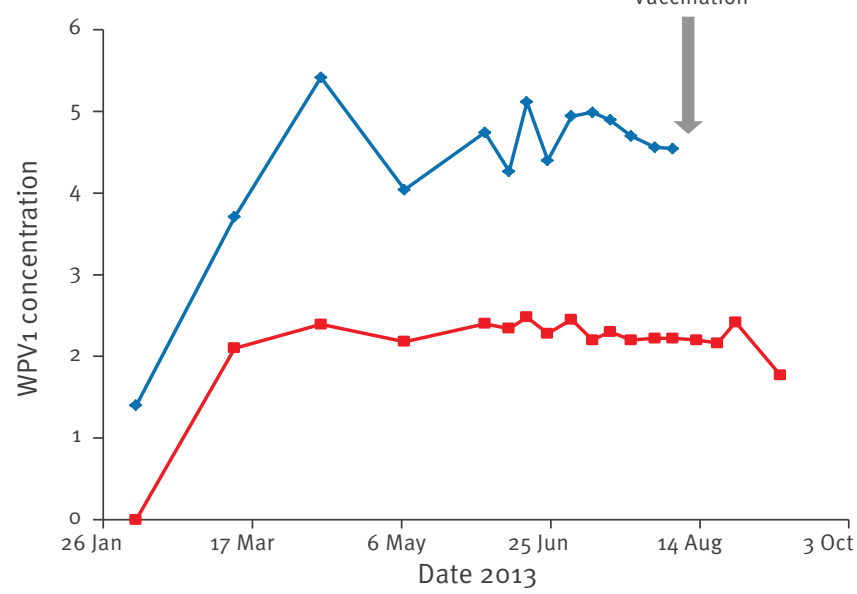

Ct: cycle threshold; pfu: plaque-forming units; q: quantitative; SOAS: South Asia; WPV1: wild poliovirus type 1.

The blue line shows the results of the plaque assay depicting the log of the calculated pfu per litre of original sewage sample. The red line shows the results of the WPV1-SOAS qRT-PCR assay depicting an inverted graph of $\mathrm{Ct}$ values representing the difference between Ct 50 (no virus particles) and the actual Ct value obtained by the test results divided by 10 . This calculation was used for a better demonstration of the similarity between the two quantitative methods, putting them on the same scale and showing the same trend. The plaque assay was discontinued after the start of the bivalent oral poliovirus vaccine supplementary immunisation campaign in early August 2013 because the assay cannot distinguish between WPV1-SOAS and the Sabin strains excreted by immunised children, while the qRTPCR assay is specific for WPV1-SOAS and was not affected by high concentrations of Sabin 1 and 3 strains in the sample [15]. Tube cultures were continued to be used to produce live WPV 1 , which was identified by standard intratypic differentiation methods and by sequence analysis.

a Three sampling sites are shown: the sewage treatment facilities of Rahat (a Bedouin city), Beer Sheva (Jewish and Bedouin communities) and Kiryat Gat (Jewish city).

b Bivalent oral polio vaccine was used in a supplementary immunisation campaign starting in early August 2013 and lasting till the end of October.

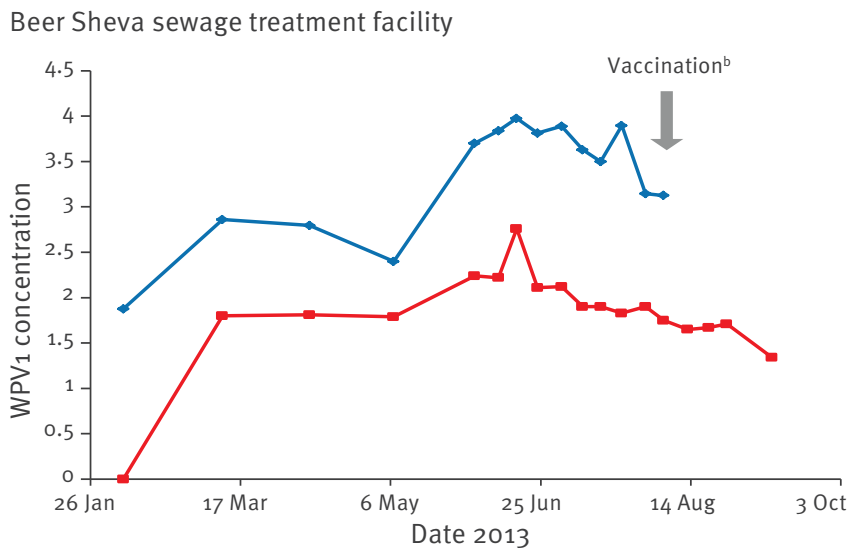

Kiryat Gat sewage treatment facility Vaccination ${ }^{b}$

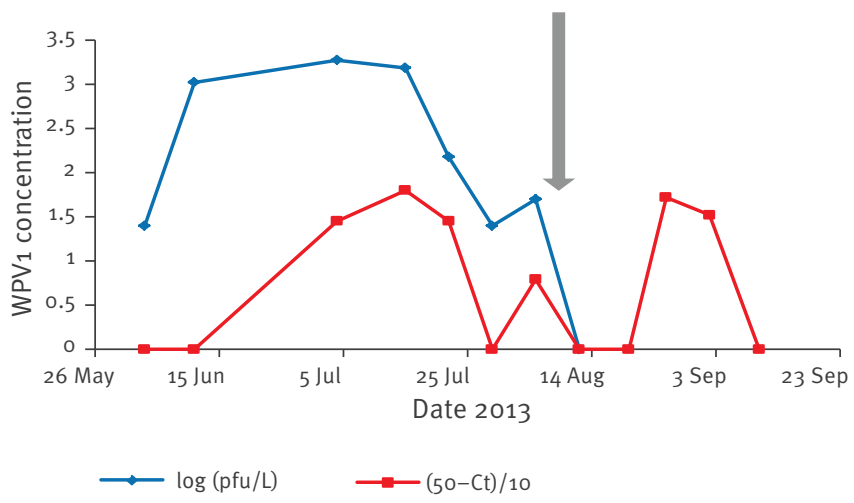


inside Beer Sheva (not shown in Figure 2), which were sampled through sewage lines upstream of Beer Sheva sewage treatment facility were negative once and were not sampled again.

The dynamics over time of the plaque assays and the WPV1-SOAS qRT-PCR Ct values in three sampling sites with distinctive characteristics are shown (Figure 3): Rahat sewage treatment facility, which collects sewage only from the Bedouin city of Rahat; the Beer Sheva sewage treatment facility, which collects sewage from Jewish cities (Beer Sheva and Ofakim) and from Bedouin communities (Tel-Sheva and Segev Shalom); and the Kiryat Gat sewage treatment facility, which collects sewage only from the Jewish city of Kiryat Gat. The plaque assay was discontinued after the beginning of the bivalent OPV (bOPV) supplementary immunisation campaign in early August 2013 [13] because of interference from the high amounts of Sabin strains excreted by immunised children, while the qRT-PCR assay was not affected by high concentrations of Sabin 1 and 3 strains [15]. Live poliovirus continued to be isolated by mass culture in L20B cells, as outlined in Figure 1.

Testing of the sites (Figure 3) reflected the epidemiological situation in southern Israel: rapid increase and persistence of high WPV1-SOAS viral load in the sewage of Beer Sheva and Rahat, indicating sustained transmission, and gradual decrease, which began in Rahat only after the beginning of the immunisation with bOPV in August, and in Beer Sheva since June. In contrast, in Kiriat Gat, WPV1-SOAS was detected intermittently and this site became negative even before bOPV vaccination began but repeatedly tested positive again afterwards. This may suggest non-persistent transmission in that area. Some of the other sites were found positive once or twice: for example, the port city

\section{FIGURE 4}

Location of sewage sampling sites in Centre (part), Tel Aviv, Haifa and North health districts in Israel with WPV1-SOAS test results, February-August 2013

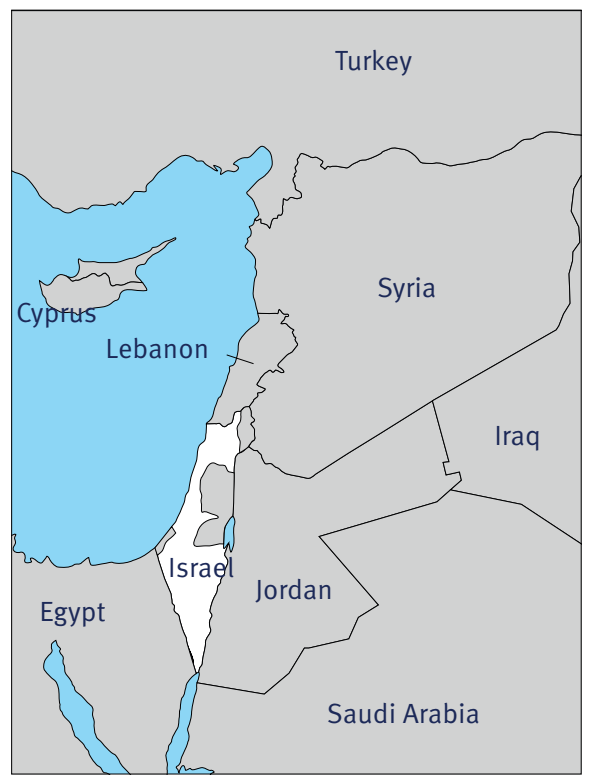

- Always positive

- Intermittently positive

$\Delta$ Negative
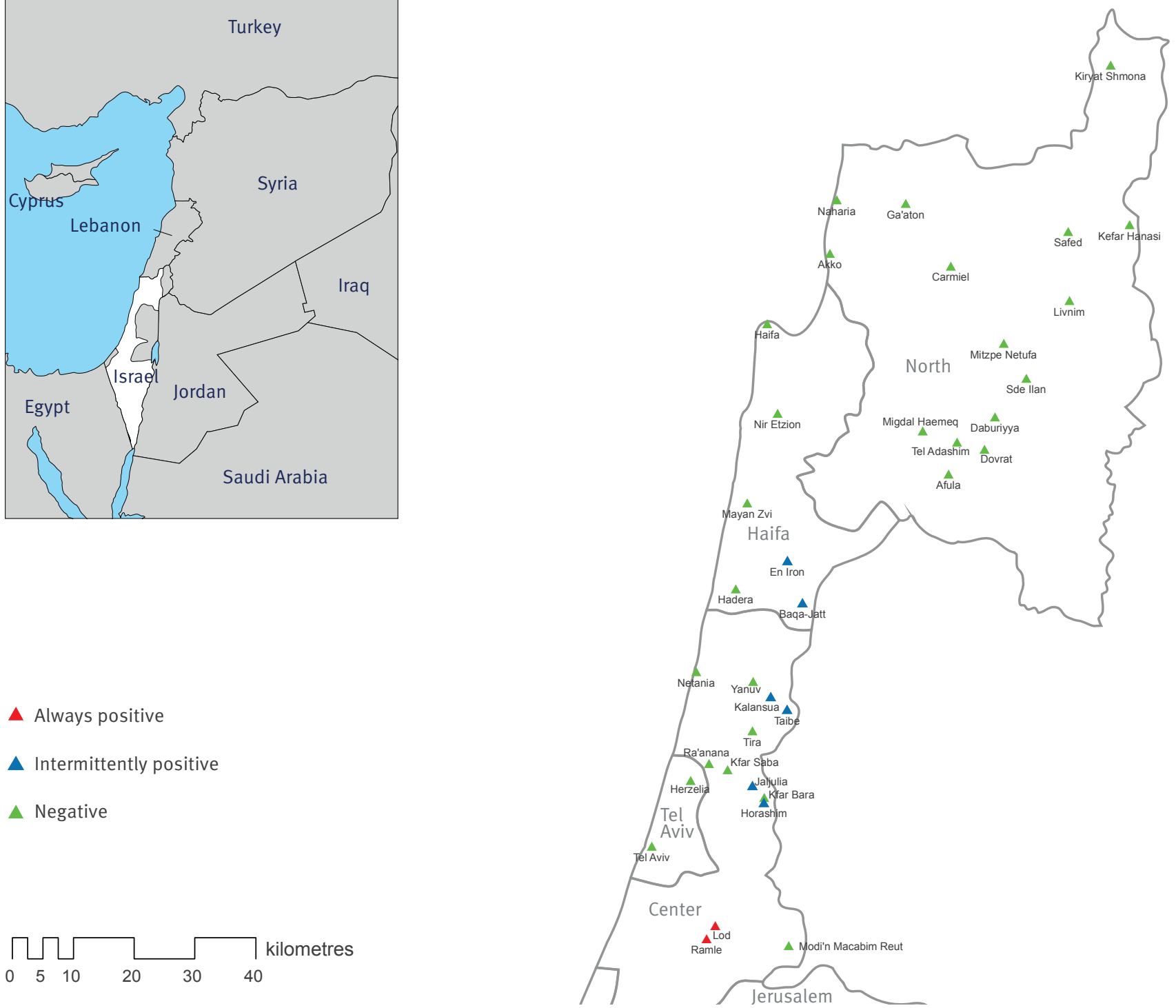

SOAS: South Asia; WPV1: wild poliovirus type 1. 
of Ashdod was found positive on 4 and 17 June and was negative in subsequent sampling in July and August.

These results indicate a high and persistent circulation of WPV 1 -SOAS in the Bedouin communities and low or no circulation in the Jewish communities, according to city of residence. Our results also demonstrate an excellent correlation between the $\mathrm{Ct}$ values of the direct qRT-PCR test and the plaque assay (Figure 3), suggesting that direct testing of processed sewage samples by qRT-PCR is a reliable approach that can be used for rapid response under emergency situations associated with WPV circulation.

\section{Spread of WPV1-SOAS to central Israel}

Towards the end of June 2013, routine surveillance in central and northern Israel was enhanced from 6 to to 46 sampling sites including sewage treatment facilities and upstream sewage lines representing major cities and populations of Israel in all five health districts: Jerusalem, Centre, Tel Aviv, Haifa and North. The sampling intervals for each site were according to the results obtained for these sites and the type of the populations. Small communities negative for WPV1-SOAS or low-risk communities without frequent contacts with populations from WPV1-SOAS-positive communities were sampled once during August. In contrast, major cities, cities positive for WPV1-SOAS or communities at high-risk due to frequent contacts with populations from WPV1-SOAS -positive communities or due to geographical proximity to other positive sites in densely populated areas were sampled several times. A total of 14 positive samples (two always positive and 12 intermittently positive) were found in three health districts: Center, Jerusalem and Haifa, while Tel Aviv and the North districts remained negative for WPV1SOAS. Much lower numbers of plaque-forming polioviruses were recovered from these sites compared with the sites covering the Bedouin cities in the South district. The geographical location and poliovirus circulation status for most of these sites is shown in Figures 2 and 4 . Nine positive sites were found in the Centre district (six are shown in Figure 4), of which only two cities, with mixed Jewish and Arab populations, were always positive (Lod and Ramle). Three intermittently positive sites were found in Jerusalem district (shown in Figure 2 as one triangle) representing Jewish and Arab mixed populations, and two intermittently positive sites were found in Haifa district (shown in Figure 4), one representing an Arab population and one representing a mixed Jewish and Arab population.

The finding of positive sewage samples outside of the Southern District provided evidence for continuous spread of the WPV1-SOAS and supported the decision to expand the supplementary immunisation activity using bOPV which began on 5 August in children in the South to children in the rest of the country (commencing 18 August).

\section{Discussion}

Our study highlights the critical role of environmental surveillance for monitoring global WPV circulation. In countries with high vaccination coverage (about 90-95\%), acute flaccid paralysis (AFP) surveillance might not detect virus introduction and circulation as occurred in Israel: enhanced AFP surveillance and aseptic meningitis surveillance implemented in response to the WPV1-SOAS detection in sewage did not identify any polio-associated illness from early June to late August [13] and later (data not shown). In contrast to previous introductions of WPV 1 to Israel and Gaza detected by the environmental surveillance [11], after which the virus disappeared without the need for supplemental immunisation (in Israel) or following national immunisation days (in Gaza) [7], in 2013 , virus importation resulted in sustained circulation [13], which eventually necessitated supplemental immunisation activity with bOPV.

The intensified environmental surveillance for polio, which was essential for management of the event, led to the development of a novel approach, implementation of a qRT-PCR assay specific to the outbreak virus. Together with modifying the testing algorithm, this allowed testing a large number of samples to be tested and result produced within a few days. The plaque and qRT-PCR assays are very precise and quantitative when used on pure viral stocks or on spiked negative sewage samples [15]. Although they are less precise when used on positive sewage samples, because of their variable contents [3], they were still very useful in assessing the intensity of virus circulation in different communities. This approach had not been used in previous outbreak investigations and allowed identification of the most affected communities and the epicentre of the silent outbreak. For example, in Rahat we continuously obtained a few hundred plaques per $\mathrm{mL}$ of concentrated sewage (after correcting for the dilution factor) while in Kiryat Gat the numbers ranged between less than 10 and up to 80 . Thus, we estimated that the number of WPV 1 excretors in Rahat may reach hundreds and may be much higher than in Kiryat Gat. These estimates were later confirmed by a stool survey that assessed the prevalence of WPV 1 excretion among subpopulations (data not shown). WPV1 circulation was probably propagated by the accumulation of a large cohort of children who had been immunised only with IPV, and not with a combination of IPV and OPV, since 2005 [13]. The qRT-PCR assay replaced the plaque assay for quantification of WPV1-SOAS excretion after the beginning of the immunisation campaign since the plaque assay was unable to distinguish between WPV1SOAS and the Sabin 1 and 3 vaccine strains found at high concentration in the sewage.

While no paralytic poliomyelitis cases were identified, the epidemiological picture that unfolded by the intensified environmental surveillance, including sample collection from sewage upstream lines, was very detailed with regard to virus circulation in different 
communities. This included persistently positive sites (mostly with a high number of plaques and corresponding low Ct values in the qRT-PCR), with sustained virus transmission rates and intermittently positive sites (lower number of plaques and higher Ct values), which indicated either a lower rate of virus transmission or occasional importations by visitors or dayworkers from Rahat (involved in various types of work), who we assume were a possible source of the virus found in the sewage. On the basis of intensive sewage surveillance using semi-quantitative methods, we speculated that the epicentre of the outbreak was in the Bedouin communities in southern Israel, in which sustained transmission has occurred. These findings have prompted an IPV catch-up campaign among Bedouin communities to minimise the already low risk for clinical poliomyelitis and to initiate bOPV supplemental immunisation to communities in southern Israel before introducing the campaign to the rest of the country.

Our report highlights the importance of environmental surveillance, which is the most sensitive and efficient approach for detection of WPV introduction and circulation in highly immunised populations. It requires systematic composite sample collection and experienced laboratories, but under these conditions, it has the highest 'population sensitivity', compared with other methods [3]. The routine environmental surveillance programme implemented in Israel since 1989 included monthly sampling of large cities and populations at high risk of virus penetration, covering around 40-50\% of the Israeli population, which now counts around 8 million people [19]. During the silent WPV 1 outbreak, coverage of the programme was increased, to around $70 \%$ of the population, focusing on communities with circulating virus. Other supplementary surveillance approaches addressing the general population rather than only AFP cases, which are currently in use in countries in Europe and elsewhere and which can detect subclinical circulation, are stool surveys or general testing of enterovirus PCR-positive stools from patients without poliomyelitis symptoms for the presence of poliovirus by culture on $\mathrm{L} 20 \mathrm{~B}$ cells. France, the Netherlands and Australia, for example, implement enterovirus surveillance that includes testing for poliovirus [20-22]. However, none of the surveillance methods used is comparable to environmental surveillance in efficiently covering large populations. For example, in France 192,598 samples were tested over five years, between 2000 and 2004 [20], which on an average yearly basis represent roughly less than $0.3 \%$ of the French population [23], while in Israel, routine surveillance before 2013 covered $30-40 \%$ of the population. Environmental surveillance could be very useful for monitoring and detecting WPV introduction and silent circulation in countries that use only IPV.

The WPV1-SOAS specific qRT-PCR assay is limited to the detection of this particular virus lineage and may lose its sensitivity even if there are minor mutations, which may occur naturally during WPV evolution. It cannot detect other poliovirus types or strains such as the WEAF lineage or type 2 vaccine-derived polioviruses (VDPV2), which circulate in Africa. Therefore, adoption of this method should take these limitations into consideration.

In conclusion, our study provides a proof of concept for the rapid implementation of $q R T-P C R$ in the framework of an outbreak in which short turnaround times and high-throughput testing are essential for incident management, while maintaining confirmatory culturebased methods. In addition, molecular assays capable of directly detecting a wider range of wild polioviruses and VDPVs currently in global circulation should be developed for routine surveillance and emergency response.

\section{Acknowledgments}

The authors are grateful to the dedicated staff of the Central Virology Laboratory, particularly Tova Halmout, Irena Agobaiev and Yuri Perepliotchikov (sample processing and virus culturing), Roberto Azar (sequencing of viral isolates), Jacklyn Alfandary and Ilana Zilberstein (molecular confirmation). We also wish to thank Michal Tepperberg, Michal Mandelboim, Liora Regev, Hilda Shaharbany, Virginia Levi and Irena Jornist for helping in sample processing and testing at times of heavy workload. We also wish to thank the South Health District Office and Environmental Health Department, particularly Yotvat Bar El (sample acquisition) and the Israel Centre for Disease Control, particularly Zalman Kaufman and Tamar Shohat (GIS maps).

\section{Conflict of interest}

None declared.

Authors' contributions

Yosef Manor: responsible for conducting the polio sewage surveillance, virus isolation and data analysis. Planned conducted and analysed results of the sewage surveillance; participated in writing of the manuscript.

Lester M Shulman: .planned the laboratory algorithm, analysed results of the sewage surveillance, supervised and conducted typing and molecular analyses, participated in writing of the manuscript.

Ehud Kaliner: was in charge of supervising the environmental surveillance activities, sampling programme and participated in data analysis.

Musa Hindiyeh: in charge of assay development and performance of clinical testing. Developed and validated the realtime RT-PCR assays for the wild poliovirus type 1 (SOAS) and for Sabin 1 and Sabin 3 strains. Conducted all the real-time RT-PCR testing and analysed the results.

Daniela Ram: participated in development and validation of the real-time RT-PCR specific assay for SOAS, Sabin 1 and Sabin 3 and, participated in testing and data analysis.

Jacob Moran Gilad: led and guided the validation of the real-time RT-PCR specific assays for SOAS, Sabin 1 and Sabin 3. Participated in data analysis and in drafting of the manuscript.

Danit Sofer: conducted virus isolations in tube cultures and participated in the validation of the sensitivity and specificity of the real-time RT-PCR specific assays and in data analysis. 
Boaz Lev: participated in supervision and evaluation of the surveillance activities and results on a national level. Itamar Grotto: participated and supervised the environmental surveillance activity in all districts, and in data analysis and manuscript preparation.

Roni Gamzu: was involved in evaluation and in routine consultations regarding the environmental surveillance sampling programme and results.

Ella Mendelson: coordinated and supervised the laboratory groups' work, participated in planning of the environmental surveillance, development and validation of the real-time RTPCR assay, and data analysis, wrote the manuscript.

\section{References}

1. Global Polio Eradication Initiative. Polio this week. Geneva: Global Polio Eradication Initiative. [Accessed 20 Feb 2014]. Available from: http://www.polioeradication.org/ Dataandmonitoring/Poliothisweek.aspx

2. Centers for Disease Control and Prevention (CDC). Laboratory surveillance for wild and vaccine-derived polioviruses worldwide, January 2008-June 2009. MMWR Morb Mortal Wkly Rep. 2009 Sep 4;58(34):950-4.

3. Hovi T, Shulman LM, van der Avoort H, Deshpande J, Roivainen $M$, de Gourville EM. Role of environmental poliovirus surveilllance in global polio eradication and beyond. Epidemiol Infect. 2012;140(1):1-13. http://dx.doi.org/10.1017/ So95026881000316X

4. Blomqvist S, El Bassioni L, El Maamoon Nasr EM, Paananen A, Kaijalainen S, Asghar H, et al. Detection of imported wild polioviruses and of vaccine-derived polioviruses by environmental surveillance in Egypt. Appl Environ Microbiol. 2012;78(15):5406-9. http://dx.doi.org/10.1128/AEM.00491-12

5. Slater PE, Orenstein WA, Morag A, Avni A, Handsher R, Green MS, et al. Poliomyelitis outbreak in Israel in 1988: a report with two commentaries. Lancet. 1990;335(8699):1192-5; discussion 1196-8. http://dx.doi.org/10.1016/0140-6736(90)92705-M

6. Shulman LM, Handsher R, Yang CF, Yang SJ, Manor J, Vonsover A, et al. Resolution of the pathways of poliovirus type 1 transmission during an outbreak. J Clin Microbiol. 2000;38(3):945-52.

7. Manor Y, Handsher R, Halmut T, Neuman M, Bobrov A, Rudich $\mathrm{H}$, et al. Detection of poliovirus circulation by environmental surveillance in the absence of clinical cases in Israel and the Palestinian authority. J Clin Microbiol. 1999;37(6):1670-5.

8. Manor Y, Blomqvist S, Sofer D, Alfandari J, Halmut T, Abramovitz B, et al. Advanced environmental surveillance and molecular analyses indicate separate importations rather than endemic circulation of wild type 1 poliovirus in Gaza district in 2002. Appl Environ Microbiol. 2007;73(18):5954-8. http:// dx.doi.org/10.1128/AEM.02537-06

9. Shulman LM, Manor Y, Sofer D, Mendelson E. Bioterrorism and surveillance for infectious diseases - lessons from poliovirus and enteric virus surveillance J Bioterr Biodef. 2012;S4.

10. Shulman LM, Manor Y, Handsher R, Delpeyroux F, McDonough MJ, Halmut T, et al. Molecular and antigenic characterization of a highly evolved derivative of the type 2 oral poliovaccine strain isolated from sewage in Israel. J Clin Microbiol. 2000;38(10):3729-34

11. Shulman LM, Manor Y, Sofer D, Handsher R, Swartz T, Delpeyroux F, et al. Neurovirulent vaccine-derived polioviruses in sewage from highly immune populations. PLoS One. 2006;1:e69. http://dx.doi.org/10.1371/journal.pone.0000069

12. World Health Organization (WHO). Poliovirus detected from environmental samples in Egypt. Geneva: WHO; 11 February 2013. Available from: http://www.who.int/csr/don/2013_02_11/ en/index.html

13. Anis E, Kopel E, Singer SR, Kaliner E, Moerman L, Moran-Gilad $\mathrm{J}$, et al. Insidious reintroduction of wild poliovirus into Israel, 2013. Euro Surveill. 2013;18(38):pii=20586.

14. Shulman LM, Hindiyeh M, Muhsen K, Cohen D, Mendelson E, Sofer D. Evaluation of four different systems for extraction of RNA from stool suspensions using MS-2 coliphage as an exogenous control for RT-PCR Inhibition. PLoS One. 2012;7(7):e39455. http://dx.doi.org/10.1371/journal. pone.0039455

15. Hindiyeh MY, Moran-Gilad J, Manor Y, Ram D, Shulman LM, Sofer D, Mendelson E. Development and validation of a real time quantitative reverse transcription-polymerase chain reaction (qRT-PCR) assay for investigation of wild poliovirus type 1-South Asian (SOAS)strain reintroduced into Israel, 2013 to 2014 . Euro Surveill. $2014 ; 19(7): \mathrm{pii}=20710$.
16. Kilpatrick DR, Yang CF, Ching K, Vincent A, Iber J, Campagnoli $R$, et al. Rapid group-, serotype-, and vaccine strainspecific identification of poliovirus isolates by real-time reverse transcription-PCR using degenerate primers and probes containing deoxyinosine residues. J Clin Microbiol. 2009;47(6):1939-41. http://dx.doi.org/10.1128/JCM.00702-09

17. Sofer D, Weil M, Hindiyeh M, Ram D, Shulman LM, Mendelson E. Human Nonpolio enteroviruses. In: Liu D, editor. Molecular detection of human viral pathogens. Boca Raton, FL: CRC Press; 2011. p. 37-52.

18. Shulman LM, Gavrilin E, Jorba J, Martin J, Burns CC, Manor Y, Moran-Gilad I, Sofer D, Hindiyeh MY, Gamzu R, Mendelson E, Grotto I, for the Genotype - Phenotype Identification (GPI) group. Molecular epidemiology of silent introduction and sustained transmission of wild poliovirus type 1, Israel, 2013. Euro Surveill. 2014;19(7):pii=20709.

19. Central Bureau of Statistics. 65th Independence Day - more than 8 million residents in the State of Israel. Jerusalem: Central Bureau of Statistics; 14 April 2013. Press release. 097/2013. Available from: http://www1.cbs.gov.il/www/ hodaot2013n/11_13_097e.pdf

20. Antona D, Lévêque N, Chomel JJ, Dubrou S, Lévy-Bruhl D, Lina B. Surveillance of enteroviruses in France, 2000-2004.Eur J Clin Microbiol Infect Dis. 2007;26(6):403-12. http://dx.doi. org/10.1007/s10096-007-0306-4

21. van der Sanden SM, Koopmans MP, van der Avoort HG. Detection of human enteroviruses and parechoviruses as part of the national enterovirus surveillance in the Netherlands, 1996-2011. Eur J Clin Microbiol Infect Dis. 2013;32(12):1525-31. http://dx.doi.org/10.1007/s10096-013-1906-9

22. Roberts J, Hobday L, Ibrahim A, Aitken T, Thorley B. Annual report of the Australian National Enterovirus Reference Laboratory 2012. Commun Dis Intell Q Rep. 2013;37(2):E97-E104.

23. World Population Statistics. France Population 2013. World Population Statistics; 21 Oct 2013. [Accessed 8 Feb 2014]. Available from: http://www.worldpopulationstatistics.com/ france-population-2013/ 